\title{
The Prognostic Values of a Novel Preoperative Inflammation-Based Score in Japanese Patients With Non-Small Cell Lung Cancer
}

\author{
Masaki Tomita ${ }^{\mathrm{a}, \mathrm{c}}$, Takanori Ayabe ${ }^{\mathrm{a}}$, Ryo Maeda ${ }^{\mathrm{a}}$, \\ Kunihide Nakamura ${ }^{a, b}$
}

\begin{abstract}
Background: Several previous researchers have investigated the prognostic value of the combinations of systemic inflammatory markers. However, the prognostic power of these systemic inflammatory markers is not identical. We aimed to establish a novel prognostic score based on systemic inflammatory markers.
\end{abstract}

Methods: Four hundred non-small cell lung cancer (NSCLC) patients who underwent surgery and were followed more than 5 years were included. Univariate and multivariate analyses were calculated by the Cox proportional hazards regression model.

Results: Among systemic inflammatory markers which were used for the previously reported indexes, preoperative serum $\mathrm{C}$-reactive protein (CRP) and body mass index (BMI) were independent prognostic markers in multivariate analysis, while serum albumin level, neutrophil to lymphocyte ratio and platelet to lymphocyte ratio were not. Based on this result, a novel score was established. Patients with both normal CRP $(<0.13 \mathrm{ng} / \mathrm{dL})$ and high BMI $\left(>20.6 \mathrm{~kg} / \mathrm{m}^{2}\right)$ were allocated a score of 0 . Patients in whom only one of these abnormalities was present were allocated a score of 1 , whilst those with both high CRP and low BMI were given a score of 2. Patients with score 0 had $84.44 \%$ of 5 -year cancer-specific survival, while patients with score 1 - 2 had a $61.88 \%$. On multivariate analysis, this novel score was an independent prognostic factor.

Conclusion: This novel score based on CRP and BMI might serve as an efficient prognostic indicator in resected NSCLC.

Keywords: Novel inflammation-based core; Non-small cell lung cancer; Surgery; Cancer-specific survival

Manuscript submitted August 1, 2019, accepted August 19, 2019

aDepartment of Thoracic and Breast Surgery, Faculty of Medicine, University of Miyazaki, Kihara 5200, Kiyotake, Miyazaki 889-1692, Japan

bDepartment of Cardiovascular Surgery, Faculty of Medicine, University of Miyazaki, Kihara 5200, Kiyotake, Miyazaki 889-1692, Japan

${ }^{\mathrm{c} C}$ Corresponding Author: Masaki Tomita, Department of Thoracic and Breast Surgery, Faculty of Medicine, University of Miyazaki, Kihara 5200, Kiyotake, Miyazaki 889-1692, Japan. Email: mtomita@med.miyazaki-u.ac.jp

doi: https://doi.org/10.14740/wjon1222

\section{Introduction}

Emerging evidence suggests that inflammatory cells are an essential component of the tumor microenvironment, and the inflammatory response serves as a crucial role in cancer development and progression and may be associated with systemic inflammation [1]. The systemic inflammatory response is associated with survival in patients with non-small cell lung cancer (NSCLC) $[2,3]$.

The systemic inflammatory response can be usually monitored using the concentration of specific serum proteins (albumin and C-reactive protein (CRP)) and a differential blood cell count (neutrophil, lymphocyte and platelet). Several previous researchers have investigated the prognostic value of the combinations of these systemic inflammatory markers including Glasgow prognostic score (GPS), neutrophil to lymphocyte ratio (NLR), platelet to lymphocyte ratio (PLR), prognostic nutritional index (PNI), systemic immune-inflammation index (SII), advance lung cancer inflammation index (ALI) and inflammatory prognostic index (IPI) [4-10]. As shown in Table 1 , these indexes consist of the serum CRP level, serum albumin level, neutrophil count, lymphocyte count, platelet count and body mass index (BMI) [4-10]. However, the prognostic power of these systemic inflammatory markers is not identical. Therefore, the purpose of this study was to investigate the more powerful prognostic inflammatory marker and establish a novel inflammatory prognostic index for NSCLC.

\section{Patients and Methods}

This retrospective study was approved by the Medical Ethics Committee of our hospital (O-0329). We examined the records of a sequential series of 400 patients with NSCLC between January 2008 and December 2013 in our center. There were 211 men and 189 women (median age: 69 years old). Patients with preoperative chemotherapy/radiotherapy or died in perioperative period, a history of inflammatory disease or active concomitant infection and small cell lung cancer were excluded from this study. The clinicopathological characteristics of these patients were summarized in Table 2. Complete blood count and routine biochemistry test, including serum CRP and serum albumin, of each patient, was applied 1 week before sur- 
Table 1. Summary of the Inflammation-Based Indexes

\begin{tabular}{|c|c|c|c|c|c|c|}
\hline & Neut & Lym & PLT & ALB & CRP & BMI \\
\hline Glasgow prognostic score (GPS) & & & & ○ & ○ & \\
\hline Neutrophil/lymphocyte ratio (NLR) & ○ & ○ & & & & \\
\hline Platelet/lymphocyte ratio (PLR) & & ○ & ○ & & & \\
\hline Prognostic nutritional index (PNI) & & O & & O & & \\
\hline Systemic immune-inflammation index (SII) & O & ○ & ○ & & & \\
\hline Advance lung cancer inflammation index (ALI) & ○ & ○ & & ○ & & ○ \\
\hline Inflammatory prognostic index (IPI) & ○ & ○ & & ○ & ○ & \\
\hline
\end{tabular}

Neut: neutrophil count; Lym: lymphocyte count; PLT: platelet count; ALB: serum albumin; CRP: C-reactive protein; BMI: body mass index.

gery. Preoperative patient' data were retrospectively extracted from the patients' medical records.

All patients in this study were staged according to the eighth edition Cancer Staging [11]. All patients routinely received the preoperative positron emission tomography (PET)/ computed tomography (CT) scans and brain magnetic resonance imaging (MRI) during staging. However, none of patients received invasive staging such as mediastinoscopy. There were 38 patients with pStage II and 55 patients with pStage III. Among these patients, 10 patients with pStage II and 31 patients with pStage III received postoperative adjuvant therapy (platinum-based chemotherapy). Other patients refused the proposed chemotherapy. The follow-up period was more than 5 years for all patients. The optimal cut-off values were determined according to previous reports [4-10, 12]. Briefly, the cut-off value of ALB, NLR, CRP, BMI and PLR was determined as $3.5 \mathrm{~g} / \mathrm{dL}, 5,0.13 \mathrm{ng} / \mathrm{dL}, 20.6$ and 170.5 , respectively. Survival curves were plotted via the Kaplan-Meier method and differences were compared using a log-rank test. Variables that were significant in univariate analyses were included in a multivariate analysis, which was performed using the Cox proportional hazards model. All reported P-values are two-sided. P-values $<0.05$ were considered statistically significant in the univariate analyses and multivariate analyses. All statistical analyses were performed using JMP software, version 12.2.0 (SAS Institute, Cary, NC, USA).

\section{Results}

As shown in Table 1, previously reported indexes were based on CRP, ALB, NLR, PLR and BMI. Firstly, we examined the prognostic power of these factors. As shown in Table 3, the univariate analysis revealed that all of CRP, ALB, NLR, PLR and BMI were related to cancer-specific survival. A multivariate analysis by Cox regression model showed that CRP and BMI were independent prognostic factors.

According to this result, we establish a novel score based on preoperative CRP and BMI. Patients with both normal CRP $(<0.13 \mathrm{ng} / \mathrm{dL})$ and high BMI $\left(>20.6 \mathrm{~kg} / \mathrm{m}^{2}\right)$ were allocated a score of 0 . Patients in whom only one of these abnormalities was present were allocated a score of 1 , whilst those with both abnormal CRP and low BMI were given a score of 2 . There were 181 patients with score 0,170 with score 1 and 49 with score 2 .
Since the number of patients with score 2 was small, patients were subdivided into two groups: score 0 and score $1-2$.

As shown in Figure 1, patients with score $1-2$ had a significantly poor 5-year cancer-specific survival compared to those with score $0(61.88 \%$ vs. $84.40 \%, \mathrm{P}<0.001)$.

By univariate analysis, gender, smoking status (current/ former vs. never), histology (adenocarcinoma vs. others), pT status (pT1 vs. pT2-3), pN status (pN0 vs. pN1-2), serum carcinoembryonic antigen (CEA) level (normal vs. high) and this novel score (score 0 vs. 1 - 2) were significantly associated with the 5-year cancer-specific survival. All seven clinicopathological characteristics were further investigated in multivariate analysis (Table 4). As also shown in Table 4, gender $(\mathrm{P}=0.001)$, histology $(\mathrm{P}<0.001), \mathrm{pN}$ status $(\mathrm{P}=0.003)$, serum CEA level $(\mathrm{P}=0.002)$ and this novel score $(\mathrm{P}=0.001)$ were independent factors in predicting overall postoperative cancerspecific survival, while smoking status and pT status were not.

\section{Discussion}

Previous studies have identified several inflammation/nutrition biomarkers as prognostic factors in patients with NSCLC [410]. In the present study, we establish a novel prognostic score based on CRP and BMI, and found that this novel score was a powerful prognostic factor in resected patients with NSCLC.

CRP is a non-specific marker of inflammation [13, 14]. High CRP level has been reported to be significantly associated with poor prognosis in NSCLC $[13,14]$. The underlying molecular mechanism by which serum CRP level is associated with a worse outcome of NSCLC is still not clear. Lee et al reported that lymphovascular invasion and pathologic tumor size are associated with preoperative serum CRP level [15]. High serum CRP level has also been associated with increased weight loss, reduced performance status, increased fatigue and decreased survival [16]. According to these findings, high serum CRP levels may predict poor survival.

Recently, nutritional assessment has been the focus of attention in patients undergoing surgery and the impact of BMI on long-term survival in patients with NSCLC requiring surgery has been reported $[17,18]$. However, the causal relationship between low BMI and poor prognosis of lung cancer is not clearly understood, and the effect of nutritional support on the surgical outcome is also unknown. Low BMI has been 
Table 2. Clinicopathological Characteristics of Patients

\begin{tabular}{|c|c|}
\hline & No. of patients \\
\hline Age & 128 \\
\hline$<65$ & 272 \\
\hline \multicolumn{2}{|l|}{$\geq 65$} \\
\hline Gender & 211 \\
\hline Male & 189 \\
\hline \multicolumn{2}{|l|}{ Female } \\
\hline Smoking status & 223 \\
\hline Current/former & 177 \\
\hline \multicolumn{2}{|l|}{ Never } \\
\hline Histology & 315 \\
\hline Adenocarcinoma & 85 \\
\hline \multicolumn{2}{|l|}{ Others } \\
\hline pStage & 307 \\
\hline I & 93 \\
\hline \multicolumn{2}{|l|}{ II-III } \\
\hline pT status & 266 \\
\hline pT1 & 134 \\
\hline \multicolumn{2}{|l|}{ pT2-3 } \\
\hline $\mathrm{pN}$ status & 339 \\
\hline pN0 & 61 \\
\hline \multicolumn{2}{|l|}{$\mathrm{pN} 1-2$} \\
\hline CEA & 292 \\
\hline Normal & 108 \\
\hline \multicolumn{2}{|l|}{ High } \\
\hline ALB & 99 \\
\hline Low & 301 \\
\hline \multicolumn{2}{|l|}{ Normal } \\
\hline NLR & 257 \\
\hline Low & 143 \\
\hline \multicolumn{2}{|l|}{ High } \\
\hline CRP & 276 \\
\hline Normal & 124 \\
\hline \multicolumn{2}{|l|}{ High } \\
\hline BMI & 138 \\
\hline Low & 262 \\
\hline \multicolumn{2}{|l|}{ High } \\
\hline PLR & 268 \\
\hline Low & 132 \\
\hline High & \\
\hline
\end{tabular}

CEA: carcinoembryonic antigen; ALB: serum albumin; NLR: neutrophil to lymphocyte ratio; CRP: C-reactive protein; BMI: body mass index; PLT: platelet to lymphocyte ratio.

reported to be associated with an impaired immune function, thereby resulting in poor prognosis [18].
Taken together, CRP and BMI were useful inflammation/ nutrition prognostic biomarkers, consistent with our results. To our knowledge, there was only one previous study that found the relationship between CRP + BMI and survival undergoing lobectomy for NSCLC [12]. They also used multivariate analysis and found that preoperative BMI and CRP were risk factors for poorer prognosis in smokers [12]. Unfortunately, they found this prognostic significance only in smokers and failed to find in non-smokers [12]. On the other hand, we found the prognostic significance of CRP and BMI, independent of smoking.

Considering NLR, we failed to find the independent prognostic significance of NLR (Table 3 ). Previously, the meta-analyses were performed to demonstrate the relationship between NLR and survival of patients with NSCLC $[5,19]$. However, the cut-off value of NLR used in each study was not consistent, ranging from 2.5 to $5.0[5,19]$. Peng et al [5] also performed a subgroup analysis based on cut-off value. They showed that compared with other subgroups, the subgroup with a cut-off of 5 had a significantly poorer survival with less heterogeneity. Therefore, we selected the cut-off value of NLR as 5. Yin et al [19] also performed a meta-analysis for NLR, and reported that a subgroup analysis with an NLR of $\geq 4$ is a more stable threshold for predicting prognosis and revealed significantly lower heterogeneity. Therefore, there is a possibility that when we selected another cut-off value, we might show other results about NLR. Peng et al [5] also reported that surgery subgroup seemed to have not a significant impact of NLR on survival compared with the chemotherapy subgroup. However, Yin et al [19] showed the prognostic significance of NLR for surgery subgroup. Thus, there is a possibility that a different cut-off value might exist for surgery subgroup and chemotherapy subgroup.

In this study, we revealed that this novel score based on CRP and BMI was an independent significant predictive factor for resected NSCLC. This score is simple, inexpensive and it does not require extra equipment. Thus, CRP and BMI should be taken into consideration in the therapeutic program for resected NSCLC. From the present results, induction and/ or adjuvant chemotherapies may have a significant role to play in NSCLC patients with high score. Therefore, the subgroup of NSCLC patients with high score could represent a reasonable study population for an induction and/or adjuvant therapy trial. Further prospective studies in this area are warranted. Unfortunately, we have no data about the postoperative serum CRP level and BMI. Therefore, we are now collecting these data, and we will report the relationship between postoperative change and patients' survival in our next study.

Our study had several limitations. It was a single-center, retrospective study with relatively small sample size. Thus, conclusions from the present study may have a bias, indicating that studies using a larger population are needed.

In conclusions, we demonstrate the prognostic significance of a novel score based on CRP and BMI in patients with resected NSCLC.

\section{Acknowledgments}

None to declare. 
Table 3. Univariate and Multivariate Analyses of Inflammation Markers

\begin{tabular}{|c|c|c|c|c|c|c|c|c|}
\hline \multirow{3}{*}{ Factor } & \multicolumn{4}{|c|}{ Univariate } & \multicolumn{4}{|c|}{ Multivariate } \\
\hline & \multirow{2}{*}{ Risk ratio } & \multirow{2}{*}{ P value } & \multicolumn{2}{|c|}{$95 \%$ CI } & \multirow{2}{*}{ Risk ratio } & \multirow{2}{*}{ P value } & \multicolumn{2}{|c|}{$95 \% \mathrm{CI}$} \\
\hline & & & Lower & Upper & & & Lower & Upper \\
\hline NLR & 2.284 & 0.019 & 1.158 & 4.053 & 1.547 & 0.217 & 0.758 & 2.889 \\
\hline CRP & 2.249 & $<0.001$ & 1.566 & 3.218 & 1.910 & 0.001 & 1.306 & 2.778 \\
\hline
\end{tabular}

Cl: confidence interval; ALB: serum albumin; NLR: neutrophil to lymphocyte ratio; CRP: C-reactive protein; BMI: body mass index; PLT: platelet to lymphocyte ratio.

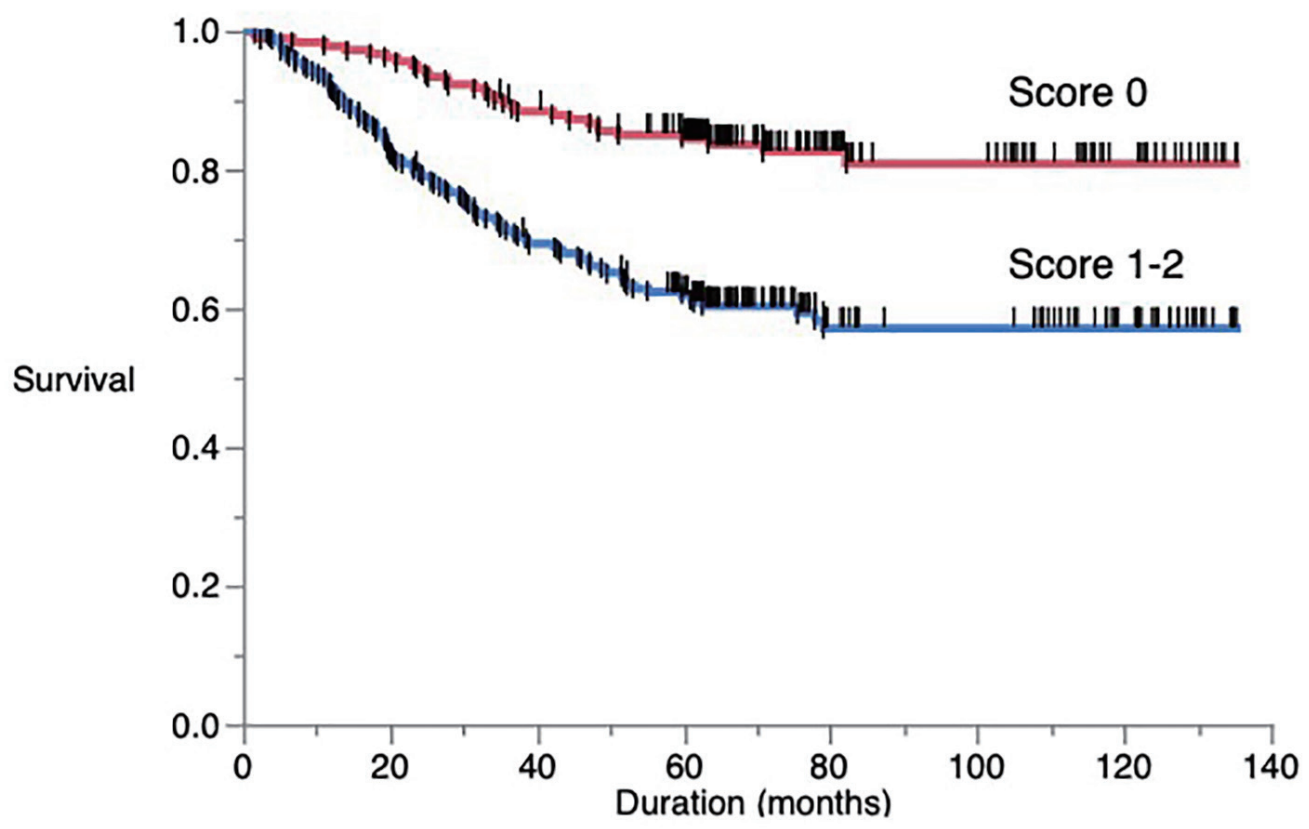

Figure 1. Overall postoperative cancer-specific survival of patients based on a novel score.

Table 4. Univariate and Multivariate Analyses

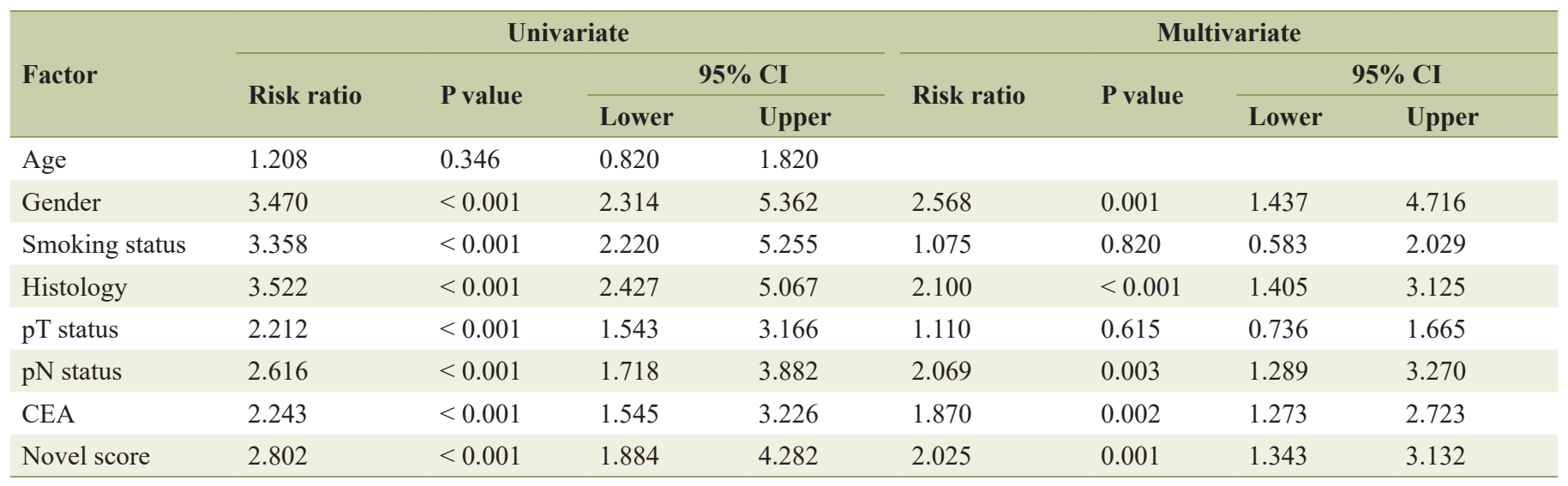

Cl: confidence interval; CEA: carcinoembryonic antigen. 


\section{Financial Disclosure}

The work was funded by the institute's intramural research funding.

\section{Conflict of Interest}

The authors have declared that no conflict of interest exists.

\section{Informed Consent}

The Medical Ethics Committee of our hospital approved the study protocol and waiver of the written informed consents from each patient, considering the retrospective design and anonymous clinical data.

\section{Author Contributions}

MT designed, performed the statistical analysis of the data and drafted the manuscript. All authors were involved in the conceptual design and review of the draft, and approved the final manuscript. KN supervised all aspects of the study.

\section{References}

1. Grivennikov SI, Greten FR, Karin M. Immunity, inflammation, and cancer. Cell. 2010;140(6):883-899.

2. Gomes M, Teixeira AL, Coelho A, Araujo A, Medeiros R. The role of inflammation in lung cancer. Adv Exp Med Biol. 2014;816:1-23.

3. Conway EM, Pikor LA, Kung SH, Hamilton MJ, Lam S, Lam WL, Bennewith KL. Macrophages, inflammation, and lung cancer. Am J Respir Crit Care Med. 2016;193(2):116-130.

4. Jin J, Hu K, Zhou Y, Li W. Prognostic value of the Glasgow prognostic score in lung cancer: evidence from 10 studies. Int J Biol Markers. 2018;33(2):201-207.

5. Peng B, Wang YH, Liu YM, Ma LX. Prognostic significance of the neutrophil to lymphocyte ratio in patients with non-small cell lung cancer: a systemic review and meta-analysis. Int J Clin Exp Med. 2015;8(3):3098-3106.

6. Ding N, Pang Z, Shen H, Ni Y, Du J, Liu Q. The prognostic value of PLR in lung cancer, a meta-analysis based on results from a large consecutive cohort. Sci Rep. 2016;6:34823.

7. Okada S, Shimada J, Kato D, Tsunezuka H, Teramukai S, Inoue $\mathrm{M}$. Clinical significance of prognostic nutritional index after surgical treatment in lung cancer. Ann Thorac Surg. 2017;104(1):296-302.

8. Zhang Y, Chen B, Wang L, Wang R, Yang X. Systemic immune-inflammation index is a promising noninvasive marker to predict survival of lung cancer: A meta-analysis. Medicine (Baltimore). 2019;98(3):e13788.

9. Tomita M, Ayabe T, Nakamura K. The advanced lung cancer inflammation index is an independent prognostic factor after surgical resection in patients with nonsmall-cell lung cancer. Interact Cardiovasc Thorac Surg. 2018;26(2):288-292.

10. Tomita M, Ayabe T, Maeda R, Nakamura K. The Inflammatory Prognostic Index Predicts Cancer-Specific Outcomes of Patients with Resected Non-Small Cell Lung Cancer. Asian Pac J Cancer Prev. 2018;19(10):2867-2870.

11. Goldstraw P, Chansky K, Crowley J, Rami-Porta R, Asamura H, Eberhardt WE, Nicholson AG, et al. The IASLC lung cancer staging project: proposals for revision of the TNM Stage Groupings in the Forthcoming (Eighth) Edition of the TNM Classification for Lung Cancer. J Thorac Oncol. 2016;11(1):39-51.

12. Nagata M, Ito H, Matsuzaki T, Furumoto H, Isaka T, Nishii $\mathrm{T}$, Yokose $\mathrm{T}$, et al. Body mass index, $\mathrm{C}$-reactive protein and survival in smokers undergoing lobectomy for lung cancerdagger. Eur J Cardiothorac Surg. 2017;51(6):11641170.

13. Jing X, Huang C, Zhou H, Li C, Fan L, Chen J, Zhang G, et al. Association between serum $\mathrm{C}$-reactive protein value and prognosis of patients with non-small cell lung cancer: a meta-analysis. Int J Clin Exp Med. 2015;8(7):1063310639.

14. Leuzzi G, Galeone C, Gisabella M, Duranti L, Taverna F, Suatoni P, Morelli D, et al. Baseline C-reactive protein level predicts survival of early-stage lung cancer: evidence from a systematic review and meta-analysis. Tumori. 2016;102(5):441-449.

15. Lee JG, Cho BC, Bae MK, Lee CY, Park IK, Kim DJ, Ahn SV, et al. Preoperative C-reactive protein levels are associated with tumor size and lymphovascular invasion in resected non-small cell lung cancer. Lung Cancer. 2009;63(1):106-110.

16. Scott HR, McMillan DC, Forrest LM, Brown DJ, McArdle CS, Milroy R. The systemic inflammatory response, weight loss, performance status and survival in patients with inoperable non-small cell lung cancer. Br J Cancer. 2002;87(3):264-267.

17. Li S, Wang Z, Huang J, Fan J, Du H, Liu L, Che G. Systematic review of prognostic roles of body mass index for patients undergoing lung cancer surgery: does the 'obesity paradox' really exist? Eur J Cardiothorac Surg. 2017;51(5):817-828.

18. Win T, Ritchie AJ, Wells FC, Laroche CM. The incidence and impact of low body mass index on patients with operable lung cancer. Clin Nutr. 2007;26(4):440-443.

19. Yin Y, Wang J, Wang X, Gu L, Pei H, Kuai S, Zhang Y, et al. Prognostic value of the neutrophil to lymphocyte ratio in lung cancer: A meta-analysis. Clinics (Sao Paulo). 2015;70(7):524-530. 